\title{
Energy Efficient Hierarchical Protocols in Wireless Sensor Networks
}

\author{
Vaishali P Bawage ${ }^{1}$, Deepak C Mehetre ${ }^{2}$ \\ ${ }^{1}$ Student - M.E. (Computer Network), K.J.College of Engineering \& Management Research, Savitribai Phule Pune University, Pune, India \\ ${ }^{2}$ H.O.D. (Computer Engineering Department), K.J.College of Engineering \& Management Research, Savitribai Phule Pune University, \\ Pune, India
}

\begin{abstract}
Wireless sensor Network comprises of a large number of sensing devices. A sensor network can be built with at least one Base station and a number of sensors. The sensor nodes are deployed on different locations, whose job is to monitor the network in real time. The monitoring and analyzing of the data that is sometimes selective parameter of same or different environment or system. In recent years, more and more sensors are deployed. Due to the limited power backup, processing capability, communication range, and these sensors should be used more efficiently since transmission and reception costs loss of energy at rapid rate. To improve the efficiency, some routing protocols have already been proposed. In this survey paper, we have reviewed various routing protocols such as LEACH, TEEN, APTEEN protocols on the basis of their functionality, complexity and energy [1]. In these protocols, the sensor nodes react not only to time critical applications but also give response to periodic intervals. The time criticality response makes the protocol an efficient protocol.
\end{abstract}

Keywords: TEEN, WSN, Energy Efficiency, Routing Protocols, Complexity

\section{Introduction}

There was a time when network solely depended on wired technology but these days the network, with ever increasing quantity of nodes and the advancement of technology can be connected wirelessly. In wireless network, nodes are distributed over planned location in any environment such as a small building, an office etc. Now-a-days, with increasing remoteness in the wireless network, sensors are deployed to monitor the nodes and transfer the information to their recipient. This type of network is known as WSN or sometimes also known as WS\&AN (Wireless Sensor \& Actuator Network). It is a distributed network of thousands of sensors of various types attached to monitor the environment conditions such as temperature, sound, vibration, etc. Most of the modern WSNs are bidirectional and also enable user to control the sensor's functionality. The functionality mainly depends on the energy consumption. The main difference between traditional networks and sensor networks is that sensors are so sensitive and small amount of energy can activate the sensors.

There are number of applications of WSNs are given below:

1) Industrial processing and monitoring.

2) Machine monitoring

3) Health care.

4) Military surveillance.

Some of the aspects of Wireless Sensor Networks are

- Node mobility

- Node heterogeneity

- Scalability

- Easy to use

- Battery operated

- Node resilience

- Cross-layer design.
Wireless sensor networks are generally battery operated devices and these batteries are non-rechargeable [1]. Wireless sensor networks are a collection of sensor node having limited resources. Sensor nodes are used to monitor physical or environmental conditions [2]. Such as temperature, pressure, sound etc. sensor node communicates the information gathered through links [2]. In wireless sensor networks the sensor nodes have a limited energy resource. Routing protocols for wireless sensor networks are responsible for the routes of the networks and have to make sure a reliable communication path. The ability of a single node is less but the total power of the entire network is enough to perform the user defined tasks. Nodes can easily be deployed in random or deterministic fashion and are normally battery operated. So energy consumption is one of the most important factor [3]. In a network there are millions of sensors which are widely and randomly deployed. The source of the energy for sensors is battery and which cannot be recharged after the deployment [4]. But the networks of the sensors are designed in such a way so that they can work up to their last. So efficiency of a network is a big and important issue in a wireless sensor network. Routing consumes a lot of energy, so an efficient and reliable routing protocol is also very important. Many protocols have been proposed in order to reduce the energy consumption in the nodes. Routing protocol have an important role in wireless sensor network because micro-sensor node dispersed in real environment field have a limitation of energy capacity, energy-efficient mechanism for wireless communication on each sensor node is so critical [5] so to increase the network life time an energy-efficient routing protocol is also necessary. For maximizing the lifetime of network, the data should be forwarded in such way that energy consumption is balanced among the nodes. Generally wireless sensor networks are made up of thousands or millions of sensor nodes. Each node have processing capabilities (microcontroller, CPUs) and may have several types of 


\section{International Journal of Science and Research (IJSR) \\ ISSN (Online): 2319-7064}

Index Copernicus Value (2013): 6.14 | Impact Factor (2015): 6.391

memory (data\& flash memory), a RF transceiver (single Omni directional antenna) a power source (batteries and solar cells) [3]. In this paper we tried to give a review on the hierarchical routing protocols. Which can help in establishing a reliable and efficient routing protocol for the wireless sensor networks.

\section{Routing Protocols for Wireless Sensor Networks}

Routing in wireless sensor network is different from routing in fixed networks in various ways. Nodes may fail. Links are variable, and routing protocols have to meet strict energy saving requirements [6] there are many routing protocols developed for wireless sensor networks. All major routing protocols for wireless sensor networks can be divided into six types as shown below in table 1 .

Table 1: Routing Protocols for Wireless Sensor Networks.

\begin{tabular}{|c|c|c|}
\hline Sr. No. & Types & Representative Protocols \\
\hline 1 & Hierarchical Protocols & $\begin{array}{c}\text { LEACH, } \\
\text { PEGASIS, } \\
\text { TEEN, }\end{array}$ \\
\hline 2 & Data - Centric Protocols & $\begin{array}{c}\text { SPIN, } \\
\text { Directed Diffusion, } \\
\text { Rumor Routing, } \\
\text { Gradient-Based Routing, } \\
\text { Energy-aware Routing. }\end{array}$ \\
\hline 3 & $\begin{array}{c}\text { Location - based } \\
\text { Protocols }\end{array}$ & $\begin{array}{c}\text { MECN, } \\
\text { SMECN, } \\
\text { GAF, GEAR, TBF. }\end{array}$ \\
\hline 4 & $\begin{array}{c}\text { Multipath - based } \\
\text { Protocols }\end{array}$ & $\begin{array}{c}\text { Sensor-Disjoint Multipath, } \\
\text { Braided Multipath, } \\
\text { N-to-1 Multipath Discovery }\end{array}$ \\
\hline 5 & $\begin{array}{c}\text { Heterogeneity - based } \\
\text { Protocols }\end{array}$ & IDSQ, CADR, CHR \\
\hline 6 & QoS based Protocols & SAR, SPEED. \\
\hline
\end{tabular}

\section{Hierarchical Protocols}

A cluster point network can reason the gateway node to become congested, particularly as the density of sensor increases. This, in turn, can cause latency in event status delivery. To permit wireless sensor networks to deal with a large population of wireless sensor networks and to cover a large area, multipoint clustering has been proposed. The goal of hierarchical routing is to manage the consumption of energy of wireless sensor network efficiently by establish the multi-hop communication within a particular cluster, and by performing data aggregation and fusion to decrease the number of transmitted packets [6].

\subsection{Low-Energy Adaptive Clustering Hierarchy (LEACH):}

W. Heinzelman et.al. [7], introduced a family of hierarchical cluster based routing protocols. A protocol which falls in this family includes cluster formation called LEACH (Low Energy Adaptive Clustering Hierarchy). LEACH is one of the first hierarchical protocols. When the node in the network fails or its battery stops working then LEACH protocol is used in the network. Leach is self-organizing, adaptive clustering protocol[3] in which nodes will arrange themselves into clusters and cluster members selects cluster head to avoid too much energy usage and incorporate data aggregation which reduces the amount of message sent to the base station. Each cluster is guided by the selected cluster head. The basic operation of LEACH is organized in two distinct phases, i.e. setup phase and steady state phase [2].

Setup phase:

- For organizing the network into clusters.

- Announcement of cluster heads

- Communication schedule creation.

Steady State phase:

- The data aggregation

- Compression

- Communication to the sink.

It reduces energy consumption by switching close (off) node heads as much as possible in LEACH all node sensors transmits directly to the cluster heads using single hop routing. $\mathrm{LEACH}$ is not applicable for the networks which are deployed in large regions. It provides scalability in the network by limiting most of the communication inside various clusters of the network [8]. The basic idea is to form clusters. The sensor nodes collectively form a cluster, based on energy strength. A Cluster Head is elected dynamically to sink the data. Having elected a cluster head. The basic idea is to form clusters. The sensor nodes collectively form a cluster, based on energy strength. A Cluster Head is elected dynamically to sink the data. Having elected a cluster head.

Leach Protocol operates in two steps:

- It is also the setup phase where election of $\mathrm{CH}$ takes place. $\mathrm{CH}$ is chosen randomly based on its energy level. This is done so as to balance energy dissipation. Under TDMA/CDMA scheme, $\mathrm{CH}$ broadcasts schedule in which sensor nodes transmit data in their respective order. When schedule is completed the total time calculated is known as frame time. Each sensor node has its local slot in the frame.

- In steady phase also known as second phase, sensor nodes transmit data to $\mathrm{CH}$. $\mathrm{CH}$ receives all the data and then after aggregation, transmits to BS. Steady phase has longer duration since its job is to manage overheads so that these overheads can be minimized. 


\section{International Journal of Science and Research (IJSR) \\ ISSN (Online): 2319-7064}

Index Copernicus Value (2013): 6.14 | Impact Factor (2015): 6.391

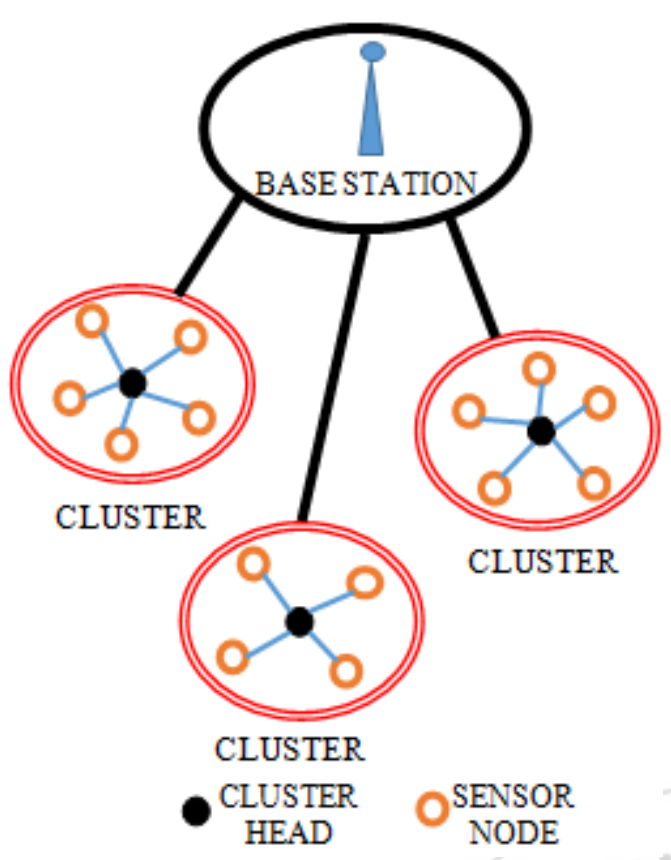

Figure 1: LEACH Protocol.

The few steps involved in PEGASIS protocol are:

- Chain Construction: Here we construct a chain by linking all the nodes. The node which is at furthermost location from the sink is selected as the starting point of the chain.

PEGASIS Protocol:

- Here, random selection occurs between the nodes, to select the leader. Leader has the ability to transmit the gathered data to the sink.

- If somehow any node in the chain dies, chain is reconstructed leaving that dead node.

- All the data from the sensor nodes is sent to the Leader/Head and that data is gathered, fused and sent to BS.

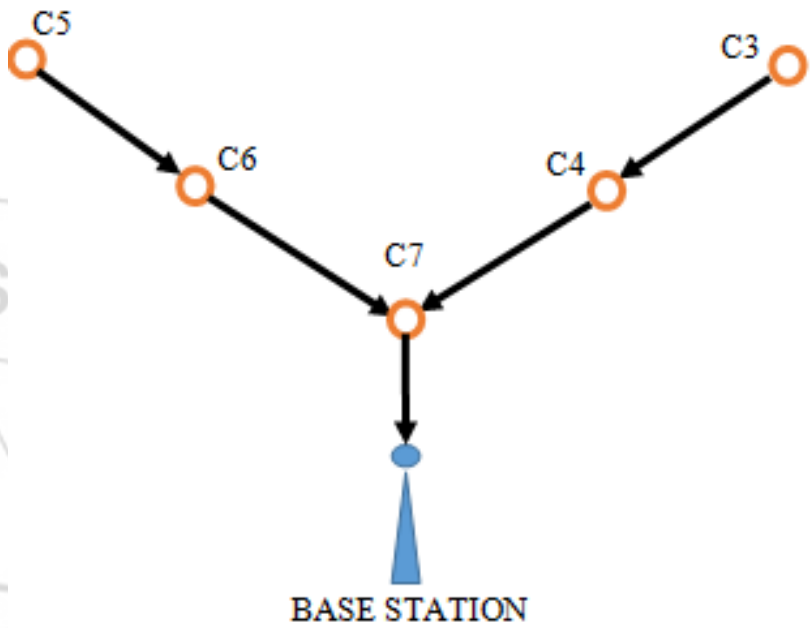

Figure 2: PEGASIS Protocol LEACH protocol by improving the cluster configuration and the delivery method of sensing data [9]. PIGASIS is an improved version of LEACH [10]. The objective of the PEGASIS is that all sensor nodes talks to each other in their neighborhood and all nodes have a spin to become cluster head. It selects one cluster-head to communicate to the base station in each round [10].

The basic operation of PEGASIS is divided into two phases:

Phase 1: Construction of chain:

Chain can be constructed by using the greedy algorithm staring from the remotest node from the base station.

Phase 2: Data gathering and Transmission to Base Station: Anyone of the node present in a network can be selected as the cluster-head randomly which communicates with the base-station. When the node is out of battery a new chain is formed bypassing the dead sensor node. The cluster head receives all the data from the other sensors and sends it to the basestation.

Here, we form a chain of nodes rather than multiple clusters. Here, we have a Base station BS, and sensor nodes. Since we do not form clusters, we do not have Cluster Heads. Each Sensor node has the ability to transmit and receive data from its neighbors. Although all sensor nodes have global knowledge which is primarily concerned with the position of each sensor node in the network. The condition of overheads is eliminated since only one node broadcasts the aggregated data. Hence, sometimes it is also called Optimal Chain Protocol.

\subsection{Threshold Sensitive} Network Protocol (TEEN)

This protocol is used in temperature sensing applications based on LEACH. TEEN is the first protocol developed for reactive networks. TEEN splits the cluster heads into the second level and uses hard-threshold and soft-threshold to detect the sudden change in network. In hard-threshold numbers of transmissions are minimized by allowing the sensor nodes to transmit only when the sensed quality is in its range. The soft threshold also minimizes the number of transmission by completely removing all the transmission. In TEEN when we are establishing or implementing the network practically we have to ensure that there are no impact or crash in the sensor node. TEEN cannot be used if we want the data regularly.

Manjeswaret. et. al. [11] proposed a protocol which is not dependent on periodic sensing of environment but senses continuously. It also belongs to the family of hierarchical clustering protocols. Nodes which are closer to each other form cluster, and transmit data to $\mathrm{CH}$ (Cluster Head). The $\mathrm{CH}$ aggregates the data and sends this data to the sink. If a second level $\mathrm{CH}$ exists, the data is first transmitted to second level $\mathrm{CH}$ and finally sinks. Here nodes sense sudden difference in the set of values and report to $\mathrm{CH}$, when there is strict need for controlling trade-off between defined parameters dynamically. These parameters can be energy efficiency, data accuracy and response time. It uses hierarchical approach with data centric methods. Also 


\section{International Journal of Science and Research (IJSR) \\ ISSN (Online): 2319-7064}

Index Copernicus Value (2013): 6.14 | Impact Factor (2015): 6.391

sensing requires less energy as compared to transmission so energy consumption is also less in this scheme. One major drawback is that while sensing application if thresholds are not reached, it cannot generate reports. $\mathrm{CH}$ sends two types of data to its neighbors, Hard Threshold and Soft Threshold.

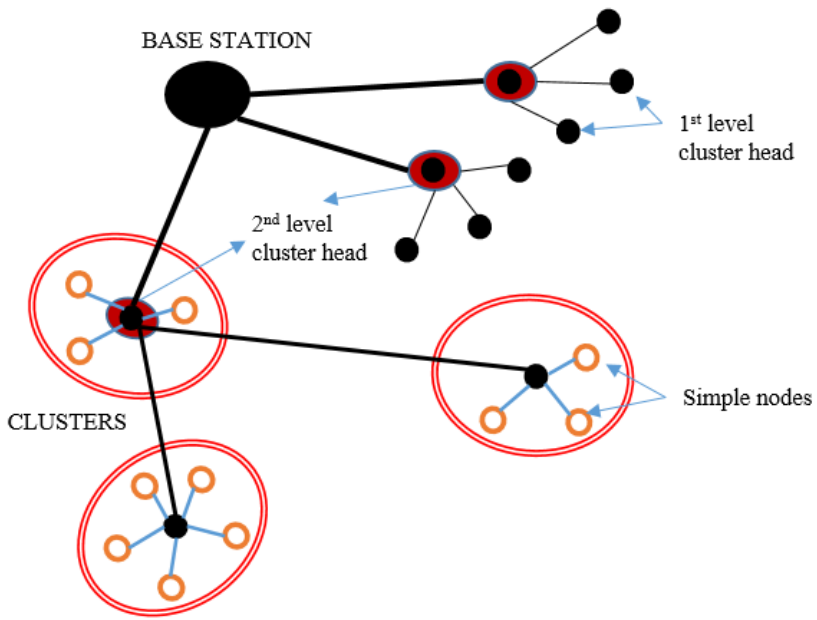

Figure 3: PEGASIS Protocol

Hard Threshold HT: It is the absolute value for the sensed attribute. The node must switch on the transmitter when the sensed value crosses the threshold and report to $\mathrm{CH}$.

Soft Threshold ST: Any small variance in the value triggers switching on of its transmitter and then transmits.

\subsection{Adaptive Threshold Sensitive Energy-Efficient Sensor Network Protocol (APTEEN)}

This protocol is also known as Adaptive TEEN. It is advancement over TEEN protocol [6]. This protocol is widely used for comprehensive information retrieval. Unlike TEEN, it not only reacts to time critical situations, but also gives idea about periodic time intervals about a particular wireless sensor network. Thus, it is a hybrid clustering protocol. It has an upper hand over previous routing protocols since we can request data in the form of persistent queries.

Example:

- Report instantly if the temperature in X Quadrant goes below $400 \mathrm{~F}$

- Retrieve the average temperature in $\mathrm{Y}$ quadrant over the last 5 hours.

APTEEN is a query based protocol which has the following types of queries.

- Historical query

- One-time query

- Persistent query

Historical query: It analyses historical data stored in the sink. (E.g. what was the pressure in $\mathrm{X}$ quadrant before 3 hours?)

One-time query: This query is used to give the overview of the network. (E.g. what was the pressure in X quadrant?)
Persistent query: This query monitors a network with respect to some parameters over a time period. (E.g. Report the pressure in $\mathrm{X}$ quadrant for the next 3 hours.)

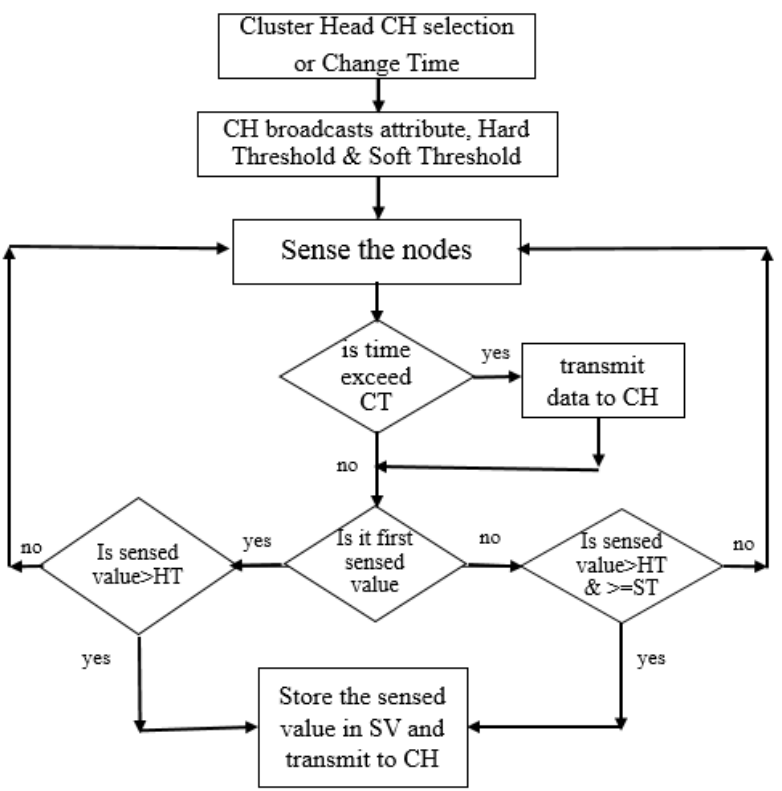

Figure 4: Operational Flow of APTEEN

\section{Conclusion}

One of the main challenges for WSN is its energy efficiency. Due to limited energy resources of sensors, it becomes a milestone to develop efficient routing protocols. One thing is that while designing routing protocol is to extent lifetime of sensor network so that sensor can operate at full probable. This reviewed and surveyed work has been done so as to focus on enhancing its energy efficiency, in a family of hierarchical routing protocols. We have covered protocols and found out that TEEN protocol has shown great response for time critical application. Its energy efficiency is good in terms of energy consumption and response time [12]. The future scope will be to make the protocols more energy efficient, so that they can with stand longer and utilize that energy more efficiently by increasing life of network.

\section{References}

[1] Dilip D. Chaudhary, Dr. L.M.Waghmare, "Energy Efficiency and Latency Improving Protocol for Wireless Sensor Networks," IEEE International Conference on Advances in Computing, Communications and Informatics, pp. 1303-1308, 2013.

[2] Ankit Solanki, Prof. Niteen B. Patel, "LEACH-SCH: An Innovative Routing Protocol for Wireless Sensor Networks," IEEE $4^{\text {th }}$ International Conference on Computing, Communications and Networking Technologies, pp. 1-5, 2013.

[3] Neha Rathi, Jyoti Saraswat, Partha Pratim Bhattacharya, "A Review on Routing Protocols for applications in wireless sensor networks," International Journal of Distributed and Parallel Systems, Vol.3, No.5,2012.

[4] Wei Bo, Hu Han-ying, Fu Wen, "An Improved LEACH Protocol for Data Gathering and Aggregation in 
Wireless Sensor Networks," IEEE International Conference on Computer and Electrical Engineering, pp. 398-401, 2008.

[5] Kee-Young Shin, Junkeun Song, Jin Won Kim, Misun Yu, Pyeong Soo Mah, "REAR: Reliable Energy Aware Routing Protocol for Wireless Sensor Networks" IEEE $9^{\text {th }} \quad$ International Conference on Advanced Communication Technology, vol. 1, pp. 525-530, 2007.

[6] Shio Kumar Singh, M.P. Singh, D.K. Singh, "Routing Protocols in Wireless Sensor Networks - A Survey," International Journal of Computer Science and Engineering Survey, Vol. 1, No. 2, 2010.

[7] W. Heinzelman, A. Chandrakasan and H. Balakrishnan, "Energy-Efficient Communication Protocol for Wireless Sensor Networks," in Proceedingls of the $33^{\text {rd }}$ Hawaii International Conference on System Sciences (HICSS'00) , 2000.

[8] Feng Sen, Qi Bing, Tang Liangrui,"An Improved Energy-Efficient PEGASIS-Based Protocol in Wireless Sensor Networks," IEEE $8^{\text {th }}$ International Conference on Fuzzy Systems and Knowledge Discovery, pp. 22302233, 2011.

[9] J. Elson and D. Estrin, "An Address-Free Architecture for Dynamic Sensor Networks," Technical Report 00274, Computer Science Department, USC, January 2000.

[10] S. Das, C. Perkins and E. Rover, "Performance Comparison of two ONdemand Routing Protocols for Ad-Hoc Networks," in Proceedings of the INFOCOM, pages 3-12, March 2000.

[11] A. Manjeshshwar and D. P. Agarwal, ”TEEN: A Routing Protocol for enhanced efficiency in Wireless Sensor Networks," 2001.

\section{Author Profile}

Vaishali P. Bawage Currently Pursing Master of Engineering in Computer Network, from K.J. College of Engineering \& Management Research, Savitribai Phule Pune University. Before that she had done Bachelor of Engineering in Computer Science and Engineering from Dr. Babasaheb Ambedkar Marathawada University.

Prof. Deepak C. Mehetre. Currently Working in KJ College of Engineering and Management Research as Head of Department for Computer Engineering Department. He has completed his Master of Engineering from Dr. Babasaheb Ambedkar Marathawada University, and currently pursing Ph.D. in Wireless Sensor Networks. 